\title{
FÍSICO-QUÍMICA EXPERIMENTAL: UMA PROPOSTA PARA ABORDAR EQUILÍBRIO DE FASES EM SISTEMAS TERNÁRIOS
}

\author{
Quelen Bulow Reiznautt e Bruna Girelli
}

Instituto de Química, Universidade Federal do Rio Grande do Sul, Av. Bento Gonçalves, 9500, Porto Alegre - RS, Brasil

Vinícius Millan Santa Catharina

Escola de Engenharia, Universidade Federal do Rio Grande do Sul, Av. Bento Gonçalves, 9500, Porto Alegre - RS, Brasil

Dimitrios Samios e Irene Teresinha Santos Garcia*

Departamento de Físico-Química, Universidade Federal do Rio Grande do Sul, Av. Bento Gonçalves, 9500, Porto Alegre - RS, Brasil

Recebido em 16/1/13; aceito em 9/5/13; publicado na web em 1/7/13

\begin{abstract}
EXPERIMENTAL PHYSICAL CHEMISTRY: A PROPOSAL TO ADDRESS PHASE EQUILIBRIA IN THREE-COMPONENT SYSTEMS. On undergraduate courses, practical classes represent periods in which students can verify the concepts presented in theoretical classes. Conversely, the teaching internship in graduate programs allows these students to incorporate pedagogical practices into their experience, predominantly involving observation and reproduction of methods adopted by supervisors. We propose internship teaching as a period for reflection on our pedagogical practice and present a methodology for an experimental physical chemistry classroom. The students can interact with the experiment and instructor, furthering the study of ternary systems while developing their skills for investigative work.
\end{abstract}

Keywords: teaching internship; experimental physical chemistry; ternary systems.

\section{INTRODUÇÃOO}

Entre os desafios apresentados na formação do químico está a melhora da qualificação para a docência dos professores universitários. ${ }^{1}$ A formação docente de professores universitários é um dos fatores importantes para a melhoria da qualidade da educação superior e um dos principais desafios é a problematização das teorias de ensino-aprendizagem nas áreas cujos professores têm formação específica não ligada aos saberes pedagógicos. A preocupação com a formação do professor para atuar em nível superior e médio fez com que o estágio de docência se tornasse obrigatório, instituído no Regulamento do Programa de Demanda Social da Coordenação de Aperfeiçoamento de Pessoal de Nível Superior (CAPES). ${ }^{2}$ Esta incorporou em seu estatuto o Estágio Supervisionado em Docência como elemento integrante da formação do pós-graduando, obrigatório para seus bolsistas de pós-graduação a partir de 2000. ${ }^{1}$ Para atender às exigências da CAPES, muitas Instituições de Ensino Superior (IES) criaram disciplinas correspondentes ao estágio de docência. Feitosa escreveu um dos primeiros artigos em que relata a problemática do estágio docência na pós-graduação e os principais debates ocorridos quando da implantação desta disciplina. ${ }^{3}$ Recentemente, com a implantação do Programa de Apoio ao Plano de Reestruturação e Expansão das Universidades Federais (Reuni) ${ }^{4}$ foram criadas as chamadas bolsas CAPES-Reuni, que promovem o desenvolvimento de atividades acadêmicas dos pós-graduandos na graduação, em colaboração com os professores responsáveis pelas disciplinas, através de auxílio à docência. Esse programa objetiva a preparação para a docência e a qualificação do ensino de graduação. O Programa de Fomento à Pós-Graduação-PROF também pode ser citado e abriu a possibilidade de realização do estágio de docência na rede oficial pública do ensino médio. ${ }^{5} \mathrm{O}$ entendimento de que os programas de pós-graduação devem contribuir à formação docente levou CAPES e $\mathrm{CNPq}$, em conjunto, a autorizarem os pós-graduandos a participarem em processos seletivos para professor substituto das IES. ${ }^{6}$

*e-mail: irene.garcia@ufrgs.br
Quadros e colaboradores ${ }^{7}$ analisaram as percepções de estudantes da pós-graduação em química e constataram que, embora $63 \%$ dos estudantes afirmassem que se sentiam preparados para lecionar na graduação, para muitos pós-graduandos, o conhecimento químico associado a qualquer experiência docente cria a sensação de preparo para a atuação como professor do Ensino Superior, mesmo que se trate de uma experiência pouco refletida e não permeada por teorias contemporâneas de ensino e aprendizagem. A prática docente deve ser um momento em que o aluno é levado a refletir sobre sua formação e a buscar articulações entre o conhecimento específico e pedagógico, entre ensinar e pesquisar, podendo também ser estimulado a refletir sobre sua prática e a propor mudanças. ${ }^{8}$

Para Maldaner e colaboradores, existe diferença entre saber os conteúdos de Química em contextos de Química e em um contexto de mediação pedagógica. ${ }^{9}$ Muito da estagnação metodológica observada nas aulas de Química dos ensinos médio e superior ocorre porque grande parte dos profissionais, principalmente no ensino superior, se formam professores sem o contexto da mediação pedagógica. A lógica de formar, somente pela observação de outro professor em sala de aula, acaba por contribuir para a estagnação no que se refere à aplicação de práticas inovadoras. Nesse sentido, seria importante que nos programas dessas disciplinas estivessem contemplados também momentos de formação pedagógica. Além disso, segundo Garcia e Kruger ${ }^{10}$ é importante o comprometimento com um ensino que propicie uma leitura Química do mundo vinculada às implicações econômicas, sociais, éticas e ambientais. Contudo, todas as iniciativas relatadas vão no sentido de contribuírem na preparação para a prática docente, ainda que ocorra no curto tempo disponível para preparo de suas teses e dissertações.

Lobo, ${ }^{11} \mathrm{em}$ pesquisa recente, mostra que o trabalho experimental, embora largamente utilizado como recurso didático nos currículos dos cursos de Química, de modo geral não tem sido adequadamente orientado no sentido de propiciar uma maior compreensão sobre a ciência Química, sua estrutura e seu processo de produção. Gonçalves e Marques analisaram artigos com propostas de experimentos em nível de graduação e pós-graduação da seção Educação da revista Química 
Nova, publicados entre 1980 e $2007 .{ }^{12}$ Observaram que a totalidade dos artigos é elaborada por docentes de Química de IES ou pós-graduandos. Os autores ainda destacaram que, entre os enfoques apresentados, os que buscaram identificar princípios ético-ambientais, como o respeito à integridade física e a preocupação com o descarte de resíduos, aspectos importantes nas atividades experimentais, enquanto que outra parte dos artigos visou propostas de experimentos simplificados e a estética dos experimentos. Naquele trabalho os autores já identificavam uma tendência de alguns autores de trazerem experimentos problematizadores e destacaram a importância da formação dos professores de ensino superior envolvidos com o discurso educacional. ${ }^{12}$

É fundamental que os professores do ensino superior possam incorporar as suas práticas, além de recursos pedagógicos inovadores, conceitos de química sustentável e de educação ambiental. Wildson e colaboradores colocam que a simples introdução do tema educação ambiental em aulas de química não é suficiente. Para isso são necessárias ações coordenadas com planejamento de projetos mais amplos com fins específicos. ${ }^{13}$ Existe a necessidade de expansão dessas áreas no meio acadêmico, como uma forma de contribuir para a formação de profissionais mais responsáveis e tecnicamente capazes na abordagem de processos químicos que incorporem a variável ambiental. ${ }^{14} \mathrm{O}$ conceito de química verde já é relativamente comum em aplicações industriais e vem, gradativamente, sendo incorporado ao meio acadêmico, no ensino e na pesquisa. Exemplos genéricos da aplicação da Química Verde em sala de aula podem incluir a substituição de um reagente tóxico por um não tóxico, redução na quantidade de solvente empregado em um processo ou aumento na eficiência energética de uma reação. ${ }^{15}$ Autores como Geris e colaboradores propuseram práticas de química orgânica com a abordagem de conteúdos relacionados à sustentabilidade tomando o biodiesel como temática. ${ }^{16}$

O trabalho aqui apresentado vai ao encontro dessas necessidades, sendo resultado das reflexões realizadas durante a prática de estágio docência. Dois de nós (QBR e ITSG), aluna do Programa de Pós-Graduação em Química da Universidade Federal do Rio Grande do Sul (UFRGS) e supervisora do estágio na disciplina de Físico-Química Experimental do Departamento de Físico-Química da UFRGS, realizamos estudos prévios e concebemos esta proposta pedagógica. Dois de nós (VMSC e BG), alunos de graduação, fomos responsáveis pelo planejamento e execução da atividade experimental e um de nós (DS), pesquisador da área de biocombustíveis, o responsável pela obtenção, uso e qualidade dos materiais utilizados. A proposta teve os seguintes objetivos:

a) proporcionar ao estudante de pós-graduação em estágio docente e ao professor da disciplina um momento para refletirem sobre o ensino de graduação, mais especificamente, o ensino experimental de físico-química;

b) a partir de uma análise crítica de um experimento abordado em aula, reescrevê-lo com metodologia que permita uma participação mais efetiva dos alunos no processo de formulação da atividade e discussão da metodologia utilizada;

c) integrar os alunos de graduação de Química à proposta para que possam, efetivamente, também serem autores da sua pesquisa, viabilizando o aprofundamento de conceitos e desenvolvendo metodologias para analisar os sistemas propostos;

d) oportunizar na atividade, ainda que parcialmente, o trabalho com conceitos que envolvam o uso de fontes renováveis e o uso da química verde no sentido de se obter uma produção mínima de resíduos.

\section{FUNDAMENTAÇÃO QUÍMICA DA ATIVIDADE PROPOSTA}

Para a atividade proposta escolheu-se um sistema baseado nos produtos obtidos na rota de síntese do biodiesel, já que nos últimos anos tem aumentado o seu uso como combustível. As motivações para o uso do biodiesel são várias, dentre elas o fato de ser biodegradável, não tóxico, produzido a partir de fontes renováveis como óleos vegetais e gorduras animais ${ }^{17} \mathrm{e}$, além disso, tem se mostrado um promissor substituto do petróleo como combustível e matéria-prima. ${ }^{18}$

Ao realizar-se uma reação de transesterificação de um triglicerídeo (óleo vegetal ou gordura) com um álcool ocorre, principalmente, a formação de glicerol e ésteres monoalquílicos, chamados biodiesel. Além desses, outros produtos como estearatos, mono e diacilgliceróis também são formados. A reação de transesterificação é reversível e, portanto, é comum o uso de álcool em excesso para garantir uma maior conversão em ésteres. ${ }^{19}$ Assim, no final da reação podemos considerar o sistema com sendo aproximadamente ternário composto, principalmente por monoalquil ésteres, glicerol e álcool, além de uma pequena quantidade de catalisador, utilizado na reação de transesterificação.

Por outro lado, um sistema de três componentes, quando monofásico, apresenta quatro graus de liberdade, que são frações molares (ou mássicas) de dois dos três componentes, temperatura e pressão. O uso de pressão e temperatura constantes reduz a variância $\left(L^{*}\right)$ do sistema e permite sua representação em um plano, de acordo com a regra das fases:

$$
\mathrm{L}^{*}=3-\varphi
$$

sendo $\varphi$ o número de fases.$^{20}$ Esses sistemas ternários são, geralmente, representados em diagramas triangulares, como triângulos retângulos e equiláteros. A representação proposta por Stokes e Roozebom faz uso de um diagrama sob a forma de um triângulo equilátero, sendo esta uma das formas mais utilizadas. ${ }^{20}$ Nesta representação, os três vértices do triângulo correspondem aos componentes puros, os lados representam sistemas binários formados pelos compostos cujos vértices são vizinhos e os pontos internos representam os sistemas ternários. A composição dos sistemas ternários é determinada com base na propriedade dos triângulos equiláteros em que a soma dos valores de qualquer reta traçada a partir de um ponto no interior do triângulo perpendicularmente a cada um dos lados, é igual à altura do triângulo.

Um sistema líquido ternário em que se desconsidera a fase vapor pode apresentar uma, duas ou três fases em equilíbrio, dependendo da miscibilidade dos componentes. A importância do estudo desses sistemas está, entre outros fatores, na previsão de formação de hidratos, nos processos industriais de purificação e extração.

Os produtos de uma reação de transesterificação podem ser parcial ou mutuamente solúveis, dependendo do tipo e composição dos reagentes e do grau de conversão. Dessa forma, dados sobre intersolubilidade dos componentes da reação são essenciais para o design de produção e para os processos de purificação dos produtos. ${ }^{21}$ Com relação à purificação dos produtos, Cerce e colaboradores ${ }^{22}$ acrescentam que o uso de catalisadores isentos de sódio ou potássio evita a formação de sabões durante a transesterificação e facilita a separação dos produtos obtidos, além de minimizar ou mesmo evitar efluentes de água de lavagem.

A decantação é um processo de separação muito utilizado na purificação de biodiesel. Embora seja uma técnica simples, é importante um conhecimento adequado do comportamento de fase do sistema biodiesel, glicerol e álcool. Dependendo das quantidades dos três componentes, pode-se obter uma separação rápida em duas fases líquidas.

Na literatura é possível encontrar alguns trabalhos envolvendo diagramas de fases de sistemas semelhantes ao proposto neste trabalho. Muitos desses trabalhos foram publicados em revistas da área de engenharia devido ao apelo tecnológico para a indústria. ${ }^{21,23-25} \mathrm{Tal}$ 
enfoque pode ser abordado nas aulas de graduação com o objetivo de encurtar a distância entre a teoria, o aprendizado experimental, a investigação e as necessidades da indústria. Na indústria de produção do biodiesel, dados de equilíbrio líquido-líquido podem ser usados para compreender os processos de separação e purificação das fases ricas em biodiesel e em glicerol. ${ }^{23}$

Trabalhos envolvendo sistemas ternários com uso de biodiesel, semelhantes ao da nossa proposta, ${ }^{21,23-29}$ são apresentados na Tabela $1 \mathrm{~S}$, disponível no Material Suplementar.

\section{FUNDAMENTAÇÃO PEDAGÓGICA APLICADA À PROPOSTA DE TRABALHO EXPERIMENTAL}

A metodologia desenvolvida neste experimento é baseada em Hodson, ${ }^{30}$ que coloca a importância da orientação do trabalho experimental para contemplar três principais aspectos da educação científica: aprender ciência (apropriação das teorias, princípios e modelos), aprender sobre a ciência (conhecimento do seu processo de produção, dos aspectos metodológicos e de validação de suas teorias) e aprender a fazer ciência (conhecimento da prática investigativa) e que pode ser trabalhada com os alunos como uma sequência de procedimentos. Sugerimos o desenvolvimento desse trabalho em quatro etapas. Essas etapas foram desenvolvidas com alunos voluntários de graduação, pertencentes aos cursos de Química Industrial e Engenharia Química.

$1^{\mathrm{a}}$. Etapa: É preparatória e inicia com a motivação à verificação de aspectos básicos envolvidos no processo de obtenção do biodiesel, tais como reagentes, produtos e subprodutos, tipos de contaminantes presentes, necessidade de separação de fases, entre outros. Esta etapa pode ser também desenvolvida interdisciplinarmente com cursos de química orgânica experimental.

$2^{a}$. Etapa: Foca na abordagem de conceitos como separação e construção de diagramas de fases.

$3^{\text {a }}$. Etapa: A investigação experimental inicia com a caracterização da miscibilidade dos três sistemas binários formados com biodiesel etílico, glicerol e etanol. Um aspecto importante a destacar é a preparação, pelos alunos, de uma ficha com os cuidados no manuseio das três substâncias e de recomendações quanto ao descarte dos resíduos.

$4^{\text {a }}$. Etapa: Os alunos devem caracterizar os diferentes sistemas ternários obtidos. Também devem propor metodologias para verificar a solubilidade relativa do etanol em cada fase, bem como relacionar esse aspecto à produção industrial de biodiesel. Os dados apresentados na "Fundamentação Química" são importantes nesta etapa. Sugerimos que o relatório tenha um formato livre para que cada grupo explore os aspectos que julgar mais importantes. A discussão mediada pelo professor, entre os grupos de alunos que realizaram as atividades, é o momento de fechamento do trabalho.

\section{DESENVOLVIMENTO DA PARTE EXPERIMENTAL}

O estudo inclui a obtenção de diagramas de fases ternários para sistema de biodiesel etílico derivado do óleo de girassol, etanol e glicerol. É importante ressaltar que nenhum roteiro prévio deve ser fornecido aos estudantes. Nesta seção são apresentados os materiais e a metodologia empregada para o experimento.

\section{Materiais utilizados}

Foram empregados o óleo de girassol refinado (Farmaquímica, Porto Alegre, Brasil), etanol (95.0\% P.A., Synth, Diadema, Brasil), hidróxido de potássio ( $98.1 \%$ pureza, Synth, Diadema, Brasil), ácido sulfúrico (98\% P.A., F. Maia, São Paulo, Brasil) e glicerol (P.A. Nuclear, Diadema, Brasil).
As densidades dos materiais usados para obtenção dos diagramas, mostradas na Tabela 1, foram obtidas experimentalmente através da pesagem de volumes conhecidos dos líquidos em balança analítica.

Tabela 1. Valores de densidade determinados experimentalmente à temperatura de $25{ }^{\circ} \mathrm{C}$

\begin{tabular}{cc}
\hline Materiais & Densidade a $25^{\circ} \mathrm{C}\left(\mathrm{g} \cdot \mathrm{mL}^{-1}\right)$ \\
\hline Biodiesel etílico do óleo de girassol & 0,857 \\
Glicerol & 1,261 \\
Etanol & 0,789 \\
\hline
\end{tabular}

\section{Síntese do biodiesel}

O biodiesel foi sintetizado partindo do óleo de girassol e usando álcool etílico como agente transesterificante, de acordo com a metodologia proposta por Guzatto et al. ${ }^{31}$ Este procedimento é uma sequiência de operações que pode ser realizada em aproximadamente 4 horas. Assim sendo, esta síntese pode ser também realizada em aulas experimentais de síntese orgânica e o biocombustível obtido ser usado para a construção do diagramas ternários em aulas experimentais de físico-química, de modo interdisciplinar.

\section{Determinação da curva binodal de solubilidade}

A curva de equilíbrio de solubilidade (curva binodal) é determinada pelo método da titulação, que consiste na observação visual da mudança de uma para duas fases (presença de turvação). O planejamento experimental, ou seja, a elaboração das tabelas com as quantidades a serem adicionadas deve abranger um largo espectro da região de miscibilidade. Partindo-se de diferentes soluções de dois dos componentes completamente miscíveis, biodiesel/etanol e glicerol/etanol adiciona-se um terceiro componente, glicerol e biodiesel, respectivamente, sob agitação vigorosa, até que o sistema apresente uma turvação característica da formação de uma nova fase. As quantidades dos componentes, no momento em que isso ocorre, definem os pontos da curva binodal que limita a zona heterogênea em equilíbrio.

No experimento desenvolvido, os sistemas binários cobriram toda a faixa de frações mássicas. Foram produzidos dez sistemas binários, os quais são mostrados na Tabela 2 e fotografados (Figura 1). A partir de frações mássicas pré-estabelecidas foram então calculadas as massas de cada componente para obter conjuntos com massa total de $20 \mathrm{~g}$. Com os valores das massas de cada componente e conhecendo-se a densidade dos mesmos podem ser calculados os volumes necessários para obtenção dos sistemas binários. As condições mantidas constantes durante o trabalho foram a temperatura de $25^{\circ} \mathrm{C}$ e a pressão atmosférica de $1 \mathrm{~atm}$.

Tabela 2. Razão percentual entre massas dos sistemas binários de partida

\begin{tabular}{lcccccc}
\hline Sistema & $\mathbf{1}$ & $\mathbf{2}$ & $\mathbf{3}$ & $\mathbf{4}$ & & \\
\hline Etanol/biodiesel & $70 / 30$ & $55 / 40$ & $35 / 60$ & $20 / 70$ & & \\
\hline Sistema & $\mathbf{5}$ & $\mathbf{6}$ & $\mathbf{7}$ & $\mathbf{8}$ & $\mathbf{9}$ & $\mathbf{1 0}$ \\
\hline Etanol/glicerol & $75 / 15$ & $70 / 30$ & $55 / 40$ & $35 / 60$ & $20 / 70$ & $10 / 70$ \\
\hline
\end{tabular}

Na Figura 1 observa-se que todos os sistemas binários obtidos (Tabela 2) se mostraram monofásicos.

A Figura 2 mostra os sistemas obtidos após adição do terceiro componente e posterior repouso por $18 \mathrm{~h}$ em recipiente fechado para garantir a separação em duas fases, sem perda de material. Os dados 


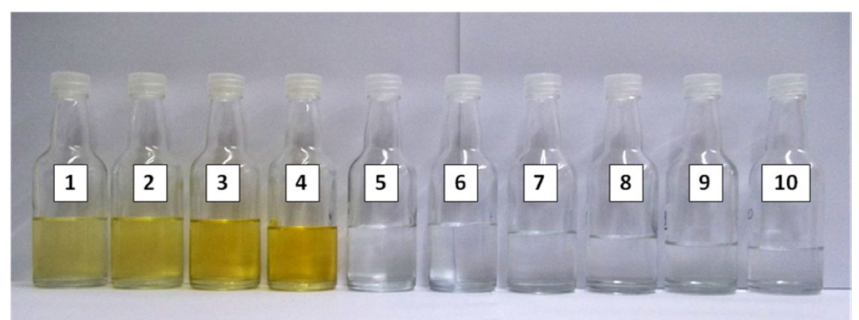

Figura 1. Sistemas binários preparados de acordo com as composições apresentadas na Tabela 2

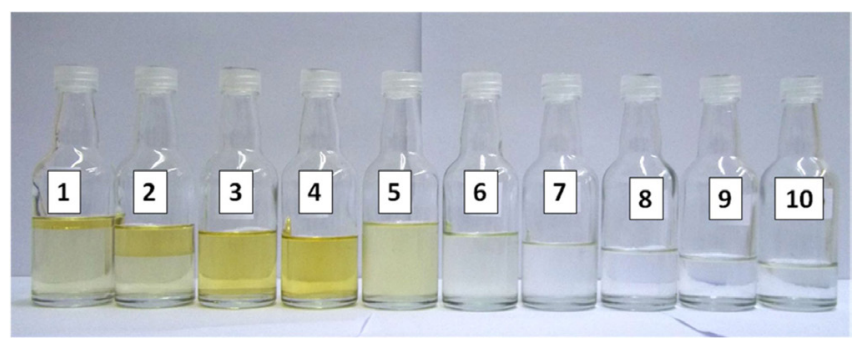

Figura 2. Sistemas ternários, compostos por biodiesel etílico do óleo de girassol, etanol e glicerol, utilizados na obtenção da zona limite de heterogeneidade, após separação de fases

primários, de volume do componente adicionado até turvação, dos sistemas 1 a 10, estão indicados na Tabela $2 \mathrm{~S}$ (material suplementar).

Devido à pequena quantidade adicionada do terceiro componente (biodiesel) necessário para obtenção de turbidez foi dificil observar a presença de duas fases (sistemas 6-10, Figura 2).

\section{Caracterização do número de fases em equilíbrio na região de turbidez}

Com o propósito de confirmar a região de heterogeneidade caracterizando o número de fases presentes, foram preparados sistemas, conforme Tabela 3. Os sistemas foram calculados com base em $20 \mathrm{~g}$, armazenados em frascos devidamente fechados, deixados em repouso por 18 horas para serem fotografados e para futura quantificação de suas diferentes fases.

Tabela 3. Frações mássicas dos sistemas ternários na região de separação de fases

\begin{tabular}{cccc}
\hline Sistema & Biodiesel & Glicerol & Etanol \\
\hline $\mathbf{1 1}$ & 0,25 & 0,50 & 0,25 \\
$\mathbf{1 2}$ & 0,25 & 0,625 & 0,125 \\
$\mathbf{1 3}$ & 0,25 & 0,25 & 0,50 \\
$\mathbf{1 4}$ & 0,50 & 0,25 & 0,25 \\
\hline
\end{tabular}

Todos os quatro sistemas ternários mostrados na Tabela 3 apresentaram rápida separação em duas fases, conforme Figura 3.

\section{Determinação das linhas de amarração}

As linhas de amarração mostram as fases que estão em equilíbrio para um determinado sistema ternário produzido. A regra das fases aplicada a sistemas ternários ( $\mathrm{P}, \mathrm{T}$ constantes) prevê que a determinação de um componente seja suficiente para caracterizar o sistema. Assim, a quantidade de etanol em cada fase nos permite obter diretamente as linhas de amarração.

Para a determinação das linhas de amarração foram obtidos três pontos dentro da zona heterogênea do diagrama ternário, mostrados

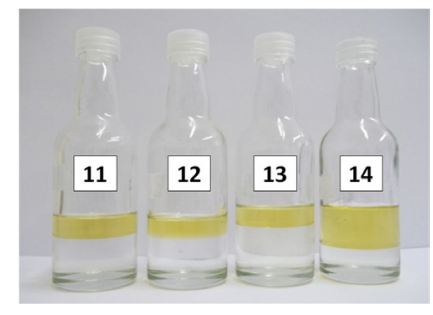

Figura 3. Sistemas ternários, compostos por biodiesel etílico do óleo de girassol, etanol e glicerol, na zona de heterogeneidade, preparados de acordo com a Tabela 3, após separação de fases

na Tabela 4, com base em $50 \mathrm{~g}$. Imediatamente após a mistura dos três componentes os sistemas se mostraram turvos. Após descanso de 18 horas observou-se separação em duas fases, conforme mostrado na figura 4. Separou-se $10 \mathrm{~mL}$ de cada uma das fases que tinham volume suficiente, as quais foram pesadas e colocadas sob agitação a $70^{\circ} \mathrm{C}$ para evaporação do etanol. Através de medidas de diferença de massa foi calculada a quantidade de etanol em cada fase. A quantidade de etanol evaporado está na Tabela 3S (material suplementar).

Tabela 4. Frações mássicas dos sistemas ternários na região de separação de fases

\begin{tabular}{cccc}
\hline Sistema & Biodiesel & Glicerol & Etanol \\
\hline A & 0,25 & 0,625 & 0,125 \\
B & 0,75 & 0,125 & 0,125 \\
C & 0,50 & 0,25 & 0,25 \\
\hline
\end{tabular}

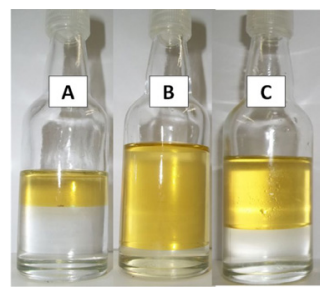

Figura 4. Sistemas utilizados para determinação das linhas de amarração, preparados conforme Tabela 4. Fotografia tomada após a separação de fase

O planejamento do experimento e escolha dos materiais definirá a duração precisa da atividade. Neste trabalho foram necessárias $3 \mathrm{~h}$ para a determinação da curva binodal de solubilidade, $2 \mathrm{~h}$ para determinação das linhas de amarração e 30 min para a caracterização do número de fases em equilíbrio na região de turbidez. Esta atividade pode ser realizada em 2 aulas de $4 \mathrm{~h}$, com um intervalo mínimo de $18 \mathrm{~h}$ entre ambas (tempo que os sistemas permanecem de repouso), para permitir a discussão dos resultados.

\section{RESULTADOS E DISCUSSÃO}

Neste estudo foi obtido o diagrama de fases para o sistema composto de biodiesel etílico derivado do óleo de girassol, etanol e glicerol.

Os 10 sistemas binários obtidos, conforme Tabela 2, são monofásicos devido à solubilidade do etanol tanto em glicerol quanto em biodiesel. Para determinação da zona de heterogeneidade, o terceiro componente foi adicionado até turvação. Observou-se também que a separação de fase, ao adicionarmos o terceiro componente, não ocorreu imediatamente. Os sistemas 11-14, Tabela 3, em menos de 15 minutos apresentaram uma interface bem definida, indicando que o tempo para visualização de fases depende da composição da mistura. 
Os três sistemas denominados A, B e C usados para a determinação das linhas de amarração se mostraram bifásicos, conforme esperado. Como colocado anteriormente, na presença de sistemas bifásicos com três componentes, em temperatura e pressão constantes, a variância é um, ou seja, apenas a determinação de um dos componentes de cada fase é necessária para caracterizar o sistema. ${ }^{20}$

A Figura 5 mostra o diagrama obtido. A diferença de densidade e de coloração entre os componentes glicerol (transparente) e biodiesel (amarelo) faz com que, através de análise qualitativa visual, seja possível presumir que uma fase é rica em glicerol (a fase mais densa) e outra rica em biodiesel (fase menos densa). Esta informação é necessária para a marcação adequada da fração de álcool na região correspondente da curva binodal.

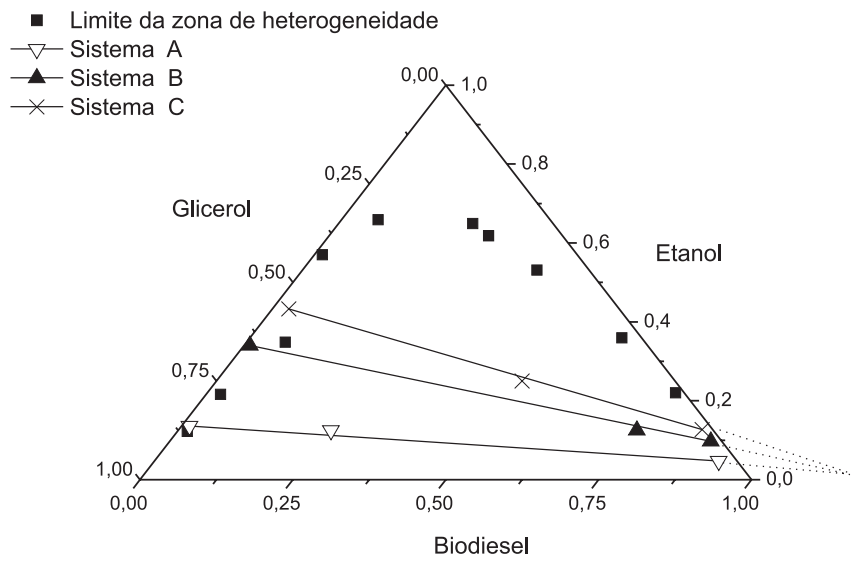

Figura 5. Diagrama ternário composto por biodiesel etílico do óleo de girassol, etanol e glicerol, mostrando as regiões mono e bifásicas à pressão de 1 atm e temperatura de $25^{\circ} \mathrm{C}$. Os sistemas $A, B$ e C, preparados de acordo com a Tabela 4 estão assinalados

É importante observar que esses resultados dependem diretamente das características do biodiesel, como tipo de cadeia, presença de contaminantes como água, sais dissolvidos, etc.

\section{CONCLUSÕES}

Neste trabalho apresentamos uma proposta que vem a contribuir para o estágio docência e pode ser também aplicada ao ensino de graduação, tendo como base referenciais teóricos bem definidos para a sua elaboração. Embora essa proposta ainda não tenha sido levada às aulas de graduação, não nos limitamos somente à orientação e aprendizado das atividades relacionadas às práticas rotineiras de laboratório. Nesta atividade pudemos ter contato com referenciais teóricos e propusemos uma abordagem diferenciada integrando à discussão pesquisadores e estudantes de graduação e pós-graduação. No experimento, a variável é o biodiesel a ser utilizado, que pode produzir resultados novos a cada experimento, dependendo da origem do óleo vegetal e das condições de síntese e purificação do mesmo. Esta variável permite, como se pode ver pelos diferentes diagramas ternários citados neste trabalho, ${ }^{21,23-29}$ que resultados diferentes sejam adquiridos nos diferentes experimentos. Esta prática faz com que os alunos não se limitem a um roteiro experimental previamente estabelecido. Como nenhum roteiro prévio foi fornecido aos estudantes, a discussão inicial com estes visa levá-los a refletir sobre a melhor forma de se obter um diagrama de fases e, consequentemente, levá-los a propor quantidades relativas de material que possam fornecer informações sobre toda a região de composição da mistura.

Além disso, trabalhamos com fontes renováveis e, no próprio experimento, na determinação das linhas de amarração, verificamos que esta atividade também pode ser utilizada para recuperar parte dos materiais utilizados, que no nosso caso foi o etanol.

A aula experimental permite criar situações próximas ao cotidiano dos alunos e a sua área de atuação, relacionando produção da matéria prima e uso desse material. Essa metodologia vai ao encontro da proposição de Hodson, ${ }^{30}$ que acreditamos ser muito válida em cursos de graduação. Entre as competências e habilidades que podem ser adquiridas com essa prática está o aprender ciência (regras de fases, elaboração de diagramas ternários e aspectos mais básicos como densidade e solubilidade), aprender sobre a ciência (relacionar conhecimentos, aprender a argumentar e a avaliar) e aprender a fazer ciência, ou seja, desenvolver habilidades investigativas e a capacidade de criticar a forma de experimentar, já que não há imposição e sim uma gama de possibilidades a serem testadas.

\section{MATERIAL SUPLEMENTAR}

As Tabelas 1S, 2S e 3S encontram-se disponíveis na forma de arquivo pdf, com acesso livre, em http://quimicanova.sbq.org.br.

A Tabela 1S contém uma revisão da literatura listando os estudos similares relacionados a diagramas ternários de sistemas semelhantes ao apresentado neste trabalho. A Tabela também mostra detalhes da forma de determinação das linhas de amarração dos diferentes sistemas.

A Tabela $2 \mathrm{~S}$ apresenta os volumes dos componentes usados para a determinação das zonas de heterogeneidade dos sistemas 1 a 10.

A Tabela 3S mostra as massas de etanol obtidas por evaporação das fases superior e inferior dos sistemas A, B e C, bem como os volumes coletados de cada fase.

\section{AGRADECIMENTOS}

Aos professores D. E. Weibel e M. O. de Souza, por oportunizarem o estágio docência no Departamento Físico-Química da UFRGS e ao CNPq (PRONEX, PIBIC/UFRGS e PIBIT/UFRGS) pelo suporte financeiro.

\section{REFERÊNCIAS}

1. de Andrade, J. B.; Cadore, S.; Vieira, P. C.; Zucco, C.; Pinto, A. C.; Quim. Nova 2004, 27, 358.

2. BRASIL, Ministério da Educação, Coordenação de Aperfeiçoamento de Pessoal de Nível Superior, Portaria no 021 de 05 de junho de 2003. CAPES, Portaria N52. In Brasil, 26 de setembro de 2002.

3. Feitosa, J. P. A.; Quim. Nova 2002, 25, 153.

4. http://reuni.mec.gov.br, acessada em Junho 2013.

5. BRASIL, Ministério de Educação, Coordenação de Aperfeiçoamento de Pessoal de Nível Superior, Portaria nº 021 de 05 de junho de 2003.

6. BRASIL, Ministério de Educação, Coordenação de Aperfeiçoamento de Pessoal de Nível Superior, Conselho Nacional de Desenvolvimento Científico e Tecnológico, Portaria conjunta CAPES/CNPq n ${ }^{\circ} 001$ de 11 de março de 2004.

7. de Quadros, A. L.; Silva, D. C.; Silva, F. C.; de Andrade, F. P.; Aleme, H. G.; Oliveira, S. R.; Silva, G. F.; Tristao, J. C.; Santos, L. J.; Quim. Nova 2011, 34, 893 .

8. Sicca, N. A. L.; Quim. Nova 1993, 16, 586.

9. Maldaner, O. A.; A formação inicial e continuada de professores de química, Unijuí: Ijuí, 2000.

10. Garcia, I. T. S.; Kruger, V.; Quim. Nova 2009, 32, 2218.

11. Lobo, S. F.; Quim. Nova 2012, 35, 430.

12. Gonçalves, F. P.; Marques, C. A.; Quim. Nova 2011, 34, 899.

13. Santos, W. L. P.; Machado, P. F. L.; Matsunaga, R. T.; Silva, E. L.; Vasconcellos, E. S.; Santana, V. R.; Rev. Eureka Enseñ. Divul. Cien. 2010, $7,260$. 
14. de Andrade, J. B.; Pinto, A. C.; Cadore, S.; Vieira, P. C.; Zucco, C.; Pardini, V. L.; Curi, L. R. L.; Quim. Nova 2005, 28, S7.

15. Lenardao, E. J.; Freitag, R. A.; Dabdoub, M. J.; Batista, A. C. F.; Silveira, C. D.; Quim. Nova 2003, 26, 123.

16. Geris, R.; dos Santos, N. A. C.; Amaral, B. A.; Maia, I. S.; Castro, V. D.; Carvalho, J. R. M.; Quim. Nova 2007, 30, 1369.

17. Reiznautt, Q. B.; Garcia, I. T. S.; Samios, D.; Mater. Sci. Eng., C. 2009, 29, 2302.

18. Follegatti-Romero, L. A.; Lanza, M.; da Silva, C. A. S.; Batista, E. A. C.; Meirelles, A. J. A.; J. Chem. Eng. Data 2010, 55, 2750.

19. Samios, D.; Pedrotti, F.; Nicolau, A.; Reiznautt, Q. B.; Martini, D. D.; Dalcin, F. M.; Fuel Process. Technol. 2009, 90, 599.

20. Pilla, L.; Físico-Química II: Equilibrio entre fases, soluções líquidas e eletroquímicas, $2^{\mathrm{a}}$. ed., UFRGS Editora: Porto Alegre, 2010.

21. Zhou, H.; Lu, H. F.; Liang, B.; J. Chem. Eng. Data 2006, 51, 1130.

22. Cerce, T.; Peter, S.; Weidner, E.; Ind. Eng. Chem. Res. 2005, 44, 9535.

23. Machado, A. B.; Ardila, Y. C.; de Oliveira, L. H.; Aznar, M.; Maciel, M. R. W.; J. Chem. Eng. Data 2011, 56, 2196.
24. Ardila, Y. C.; Pinto, G. M. F.; Machado, A. B.; Maciel, M. R. W.; J. Chem. Eng. Data 2010, 55, 4592.

25. Follegatti-Romero, L. A.; Lanza, M.; Batista, F. R. M.; Batista, E. A. C.; Oliveira, M. B.; Coutinho, J. A. P.; Meirelles, A. J. A.; Ind. Eng. Chem. Res. 2010, 49, 12613.

26. Csernica, S. N.; Hsu, J. T.; Ind. Eng. Chem. Res. 2011, 50, 1012.

27. Franca, B. B.; Pinto, F. M.; Pessoa, F. L. P.; Uller, A. M. C.; J. Chem. Eng. Data 2009, 54, 2359.

28. Negi, D. S.; Sobotka, F.; Kimmel, T.; Wozny, G.; Schomacker, R.; Ind. Eng. Chem. Res. 2006, 45, 3693.

29. Andreatta, A. E.; Casas, L. M.; Hegel, P.; Bottini, S. B.; Brignole, E. A.; Ind. Eng. Chem. Res. 2008, 47, 5157.

30. Hodson, D.; Int. J. Sci. Ed. 1992, 14, 541.

31. Guzatto, R.; Defferrari, D.; Reiznautt, Q. B.; Cadore, I. R.; Samios, D.; Fuel 2012, 92, 197. 